\title{
A New Geometric Average Technique to Solve Multi-Objective Linear Fractional Programming Problem and Comparison with New Arithmetic Average Technique
}

\author{
Samsun Nahar ${ }^{1}$, Md. Abdul Alim² \\ ${ }^{1}$ Department of Basic Sciences and Humanities, University of Asia Pacific \\ ${ }^{2}$ Department of Mathematics, Bangladesh University of Engineering and Technology \\ Dhaka-1000, Bangladesh
}

\begin{abstract}
In this paper, we have suggested a new technique to solve a MOLFPP by using new geometric averaging method. An algorithm is suggested for its solution. We have used some other techniques such as arithmetic averaging, geometric averaging, and new arithmetic averaging method to solve the same problem. New geometric averaging method gives better result than all those methods.
\end{abstract}

Keywords: Linear Fractional Programming Problem, Statistical average method, Arithmetic average, geometric average

\section{Introduction}

Linear programming is comparatively a recent mathematical concept. A study of multi-objective linear programming problem (MOLPP) is introduced in [1] which suggests an approach to set up multi-objective function (MOF) under the limitation so that the optimum value of individual problem was greater than zero. Using mean and median the MOF was studied by solving multi-objective programming problem [2]. The multiobjective linear fractional programming problem (MOLFPP) was solved by the technique developed by Chandra Sen. The industrial production planning, financial and corporate planning, healthcare and hospital planning are important fields which use linear fraction maximum problems. So it has attracted considerable research and interest. There are several methods to solve these problems discussed in [3] where linear fractional programming is transformed to an equivalent linear program. Sing conducted a useful study about the optimality condition in fractional programming [4]. In [5], Sulaiman and Othman studied optimal transformation technique to solve MOLFPP. In [6], Hamad Amin studied MOLPP using Arithmetic average. The study in [7] suggested a new technique to transform MOLPP to the single objective linear programming problem by using harmonic mean for values of functions.

In this paper I have defined a MOLFPP and suggested a new geometric average technique to optimize the objective function where a single objective function is developed from multi-objective functions. The result is compared with that of optimization using new arithmetic average technique. The results are also compared with those of optimization which are obtained by using arithmetic mean and geometric mean. This new geometric average technique gives better result than all those results.

\section{Problem Formulation}

The main objective of this study is to solve multi-objective linear fractional programming problems. Before going to this problem, I would like to discuss some common definitions which will be used to understand the target of this paper.

\subsection{Common Definitions:}

\subsubsection{Linear Programming:}

Linear Programming deals with the optimization of a function of variables known as objective function, subject to set of linear equalities/inequalities known as constraints. The objective function may be profit, loss, cost, production capacity or any other measure of effectiveness which is to be obtained in the best possible or optimal manner. G.B. Dantzig in 1947 proposed the simplex algorithm as an efficient method to solve a linear programming problem [8].

$$
\begin{aligned}
\qquad \operatorname{Max}(\min ) \quad c^{t} \vec{x} \\
\text { A linear program is of the form: } \begin{aligned}
s / t \quad & A \vec{x}=\vec{b} \\
\vec{x} & \geq 0
\end{aligned}
\end{aligned}
$$


Where, $\boldsymbol{b}$ is m-dimensional vector of constants, $\boldsymbol{x}$ is n-dimensional vector of decision variables and $\boldsymbol{A}$ is $m \times n$ matrix of constants.

\subsubsection{Simplex Method:}

The simplex method is developed using fundamental theorem of linear programming. This method sets up an algorithm which involves repetitive application of predetermined operation [9]. There are many developed methods to solve problems relating linear programming. Among them, the simplex method provides optimum solution to the problems. To find out an optimum solution using this method, the problems should be expressed in terms of linear objective function which is subject to predefined constraints [10].

\subsubsection{The Simplex Algorithm:}

In constraint based solution of any linear programming problem, the optimum results of it can be predefined. Using repetitive algorithm, the results of the linear programming problems are to be found out until an optimum solution to be reached. For this purpose, the simplex algorithm is most promising. In this algorithm, an initial value of the solution is approximated. Based on this, a nearest solution point is targeted. This process is continued until an optimum solution to be obtained [10].

\subsubsection{The Arithmetic Mean (A.M.):}

A.M. which is sometimes referred to as simply mean, is the most commonly used central value of a distribution. The A.M. is calculated by totaling the results of all the observations and dividing this total by the number of observations when the data at hand are numerical.

If the ages of six school children are $16,18,17,15,17$ and 16 years, the mean

is $\frac{16+18+17+15+17+16}{6}=16.5$ years.

\subsubsection{The Geometric Mean (G.M.):}

The geometric mean of $\mathrm{n}$ positive values $x_{1}, x_{2}, \cdots x_{n}$ is defined as the nth positive root of the product of the values. Symbolically,

G.M. $=\left(x_{1} x_{2} x_{3} \cdots x_{n}\right)^{1 / n}$; for 2 values, $x_{1}, x_{2}, G \cdot M .=\left(x_{1} x_{2}\right)^{1 / 2}$;for 3

values $x_{1}, x_{2}, x_{3}, G \cdot M .=\left(x_{1} x_{2} x_{3}\right)^{1 / 3}$

\subsection{MOLFPP:} defined as,

Multi-objective function that are the ratio of two linear objective functions are said to be MOLFPP,

$$
\begin{aligned}
& \max z_{1}=\frac{c_{1}^{t} x+\gamma_{1}}{d_{1}^{t} x+\beta_{1}} \\
& \max z_{2}=\frac{c_{2}^{t} x+\gamma_{2}}{d_{2}^{t} x+\beta_{2}} \\
& \ldots \ldots \ldots \ldots \ldots \ldots \ldots \ldots \ldots \ldots \ldots \\
& \max z_{r}=\frac{c_{r}^{t} x+\gamma_{r}}{d_{r}^{t} x+\beta_{r}} \\
& \min z_{r+1}=\frac{c_{r+1}^{t} x+\gamma_{r+1}}{d_{r+1}^{t} x+\beta_{r+1}} \\
& \ldots \ldots \ldots \ldots \\
& \min z_{s}=\frac{c_{s}^{t} x+\gamma_{s}}{d_{s}^{t} x+\beta_{s}}
\end{aligned}
$$

where, $\boldsymbol{b}$ is m-dimensional vector of constants, $\boldsymbol{x}$ is n-dimensional vector of decision variables and $\boldsymbol{A}$ is $m \times n$ matrix of constants. Both types of objective functions must be present. 


\section{MOLFPP Solution Techniques}

We can solve MOLFPP using Chandra Sen's technique but it gives comparatively poor result of the objective function. But statistical average techniques (arithmetic and geometric) which are proposed in this paper for solving MOLFPP give better result of the objective function. These techniques are briefly described below.

\subsection{Solving MOLFPP by Chandra Sen's Technique:}

We obtain a single value corresponding to each of the objective functions of MOLFPP of equation (1.1). They are being optimized individually subject to the constraints of equation (1.1) as follows:

$$
\begin{aligned}
& \operatorname{Max} z_{1}=\varphi_{1} \\
& \operatorname{Max} z_{2}=\varphi_{2} \\
& \ldots \ldots \ldots \ldots \ldots \ldots \\
& \operatorname{Max} z_{r}=\varphi_{r} \\
& \operatorname{Min} z_{r+1}=\varphi_{r+1} \\
& \ldots \ldots \ldots \cdots \cdots \cdots \\
& \operatorname{Min} z_{s}=\varphi_{s} ;
\end{aligned}
$$

where $\varphi_{1}, \varphi_{2}, \cdots \cdots \varphi_{s}$ are values of the objective functions. These values can be put in the equation (1.3), which is known as Chandra Sen's method, to find out a single objective function.

$$
\max \quad z=\sum_{i=1}^{r} \frac{z_{i}}{\left|\varphi_{i}\right|}-\sum_{i=r+1}^{s} \frac{z_{i}}{\left|\varphi_{i}\right|}
$$

where, $\varphi_{i} \neq 0, \quad i=1,2, \cdots s$. Subject to the constraints of equation (1.1) and the optimum value of the objective functions $\varphi_{i}$ may be positive or negative.

\subsection{Proposed Technique}

1.2.1 Statistical averaging method:

$$
\begin{aligned}
& \max \quad z=\sum_{i=1}^{r} \frac{z_{i}}{A \cdot M \cdot\left(A A_{i}\right)}-\sum_{i=r+1}^{s} \frac{z_{i}}{A \cdot M \cdot\left(A L_{i}\right)} \\
& \max \quad z=\sum_{i=1}^{r} \frac{z_{i}}{G \cdot M \cdot\left(A A_{i}\right)}-\sum_{i=r+1}^{s} \frac{z_{i}}{G \cdot M \cdot\left(A L_{i}\right)}
\end{aligned}
$$

Where, $A A_{i}=\left|\varphi_{i}\right|, i=1 \cdots r$ and $A L_{i}=\left|\varphi_{i}\right|, i=1+r \cdots s$

1.2.2 Solving MOLFPP by using the new arithmetic average technique:

Let $m_{1}=\min \left\langle A A_{i}\right\rangle$, where $A L_{i}=\left|\varphi_{i}\right|, \varphi_{i}$ is maximum value of $z_{i}, i=1 \cdots r$

$m_{2}=\min \left\langle A L_{i}\right\rangle$, where $A A_{i}=\left|\varphi_{i}\right|, \varphi_{i}$ is minimum value of $z_{i}, i=r+1 \cdots s$

$A . A v=\frac{m_{1}+m_{2}}{2}$ so

$\max \quad z=\left(\sum_{i=1}^{r} z_{i}-\sum_{i=r+1}^{s} z_{i}\right) / A . A v$

1.2.3 Solving MOLFPP by using the new geometric average technique:

Using $m_{1}$ and $m_{2}$ obtained in section 1.2.2, we can find the geometric average as follows:

$$
\begin{aligned}
& G . A v=\sqrt{m_{1} m_{2}} \\
& \max \quad z=\left(\sum_{i=1}^{r} z_{i}-\sum_{i=r+1}^{s} z_{i}\right) / G . A v
\end{aligned}
$$


1.3 Algorithm for new Arithmetic and Geometric average technique:

Step 1: Find the value of each of individual objective functions which is to be maximized or minimized.

Step 2: Solve the first objective problem by simplex method.

Step 3: Check the feasibility of the solution in step 2. If it is feasible then go to step 4. Otherwise, use dual simplex method to remove infeasibility.

Step 4: Assign a name to the optimum value of the first objective function $z_{1}$ say $\varphi_{1}$.

Step5: Repeat the step 2, $\mathrm{i}=1,2, \ldots \mathrm{s}$

Step 6: Select $m_{1}=\min \left\langle A A_{i}\right\rangle, m_{2}=\min \left\langle A L_{i}\right\rangle, i=1 \cdots s$

$$
A . A v=\frac{m_{1}+m_{2}}{2} \quad \text { and } \quad G . A v=\sqrt{m_{1} m_{2}}
$$

Step 7: Optimize the combined objective function with the same constraints

$$
\max \quad z=\left(\sum_{i=1}^{r} z_{i}-\sum_{i=r+1}^{s} z_{i}\right) / A . A v \quad \text { and } \quad \max \quad z=\left(\sum_{i=1}^{r} z_{i}-\sum_{i=r+1}^{s} z_{i}\right) / G . A v
$$

1.4 Program solution for new Arithmetic and Geometric average technique:

The following program can be used to solve MOLFPP by proposed method.

For this, let

$\varphi A_{i}=$ value of objective functions which is to be maximized.

$\varphi L_{i}=$ value of objective functions which is to be minimized

So

$$
\begin{aligned}
& A A_{i}=\left|\varphi A_{i}\right| ; \forall i=1 \cdots r ; A L_{i}=\left|\varphi L_{i}\right| ; \forall i=1+r \cdots s \\
& S M=\sum_{i=1}^{r} z_{i} ; \quad S N=\sum_{i=r+1}^{s} z_{i} \\
& m_{1}=\min \left\langle A A_{i}\right\rangle ; \quad m_{2}=\min \left\langle A L_{i}\right\rangle \\
& \max \quad z=(S M-S N) / A \cdot A v \text { and } \\
& \max \quad z=(S M-S N) / G \cdot A v
\end{aligned}
$$

1.5 Flow Chart for new Arithmetic and Geometric average technique:

The flow chart, shown in Fig. 1.1, describes how the objective functions are optimized.

\section{Test Calculations}

Example: Consider the following multi-objective linear fractional programming problems. But as these functions are fractional, we have to use modified simplex method first to obtain the values of these objective functions. Then we can use these values to develop a single objective function using Chandra Sen's technique. Multi-objective fractional functions:

$$
\begin{array}{llr}
\max & z_{1}=\frac{3 x_{1}-2 x_{2}}{x_{1}+x_{2}+1} & \\
\max & z_{2}=\frac{9 x_{1}+3 x_{2}}{x_{1}+x_{2}+1} & x_{1}+x_{2} \leq 2 \\
\max & z_{3}=\frac{3 x_{1}-5 x_{2}}{2 x_{1}+2 x_{2}+2} & \text { s/t } 9 x_{1}+x_{2} \leq 9 \\
\min \quad z_{4} & =\frac{-6 x_{1}+2 x_{2}}{2 x_{1}+2 x_{2}+2} & x_{1}, x_{2} \geq 0 \\
\min & z_{5}=\frac{-3 x_{1}-x_{2}}{x_{1}+x_{2}+1} &
\end{array}
$$




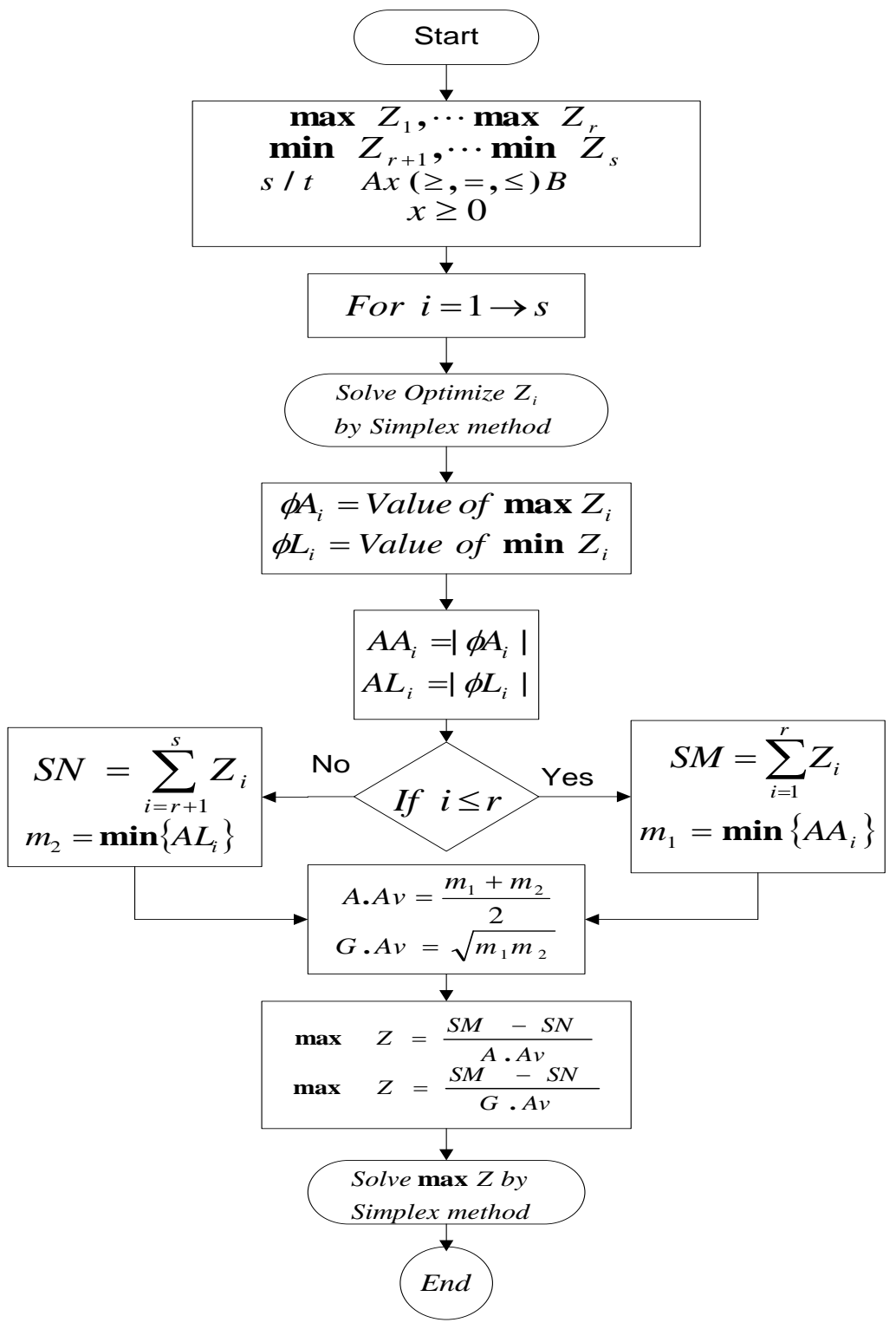

Fig. 1.1 Flow chart.

We have to solve by modified simplex method (equation (1.9)) [11] which implies

$$
\begin{array}{ll}
\max z=\frac{c x+\alpha}{d x+\beta} \quad \mathrm{s} / \mathrm{t} \quad & A x(\leq,=, \geq) b \\
& b \geq 0
\end{array}
$$

The objective function $H(y)=I y+j$

Where, $I=\frac{c \beta-d \alpha}{\beta}, y=\frac{x}{d x+\beta}, j=\frac{\alpha}{\beta} ; K y \leq L, K=A \beta+b d, L=b$

Now from equation (1.8)

$$
\max z_{1}=\frac{3 x_{1}-2 x_{2}}{x_{1}+x_{2}+1} \quad \begin{gathered}
x_{1}+x_{2} \leq 2 \\
\mathrm{~s} / \mathrm{t} 9 x_{1}+x_{2} \leq 9 \\
x_{1}, x_{2} \geq 0
\end{gathered}
$$

$c=(3,-2), d=(1,1), \alpha=0, \beta=-1, A_{1}=(1,1), A_{2}=(9,1), b_{1}=2, b_{2}=9$ 
$\max \quad H(y)=[I y]+j=\frac{c \beta-d \alpha}{\beta}[y]=(3,-2) \cdot\left[\begin{array}{l}y_{1} \\ y_{2}\end{array}\right]=3 y_{1}-2 y_{2}$

For first constraint,

$$
K_{1}=(1,1) \cdot 1+2(1,1)=(1,1)+(2,2)=(3,3), K y \leq L \Rightarrow(3,3)\left(\begin{array}{l}
y_{1} \\
y_{2}
\end{array}\right) \leq 2 \Rightarrow 3 y_{1}+3 y_{2} \leq 2
$$

For second constraint,

$$
\begin{aligned}
& K_{2}=(9,1) \cdot 1+9(1,1)=(9,1)+(9,9)=(18,10), K y \leq L \Rightarrow(18,10)\left(\begin{array}{l}
y_{1} \\
y_{2}
\end{array}\right) \leq 9 \Rightarrow 18 y_{1}+10 y_{2} \leq 9 \\
& \max , H(y)=3 y_{1}-2 y_{2} \quad \max H=3 y_{1}-2 y_{2} \\
& s / t 3 y_{1}+3 y_{2} \leq 2 \Leftrightarrow s / t 3 y_{1}+3 y_{2}+s_{1}=2 \\
& 18 y_{1}+10 y_{2} \leq 9 \Leftrightarrow \quad 18 y_{1}+10 y_{2}+s_{2}=9 \\
& y_{1}, y_{2} \geq 0 \quad \text { where } y_{1}, y_{2}, s_{1}, s_{2} \geq 0
\end{aligned}
$$

\begin{tabular}{|c|c|c|c|c|c|c|c|}
\hline \multirow[t]{2}{*}{$c_{B}$} & \multirow{2}{*}{ Basis ${ }^{c_{j}}$} & 3 & -2 & 0 & 0 & & \\
\hline & & $\mathrm{Y}_{1}$ & $\mathrm{Y}_{2}$ & $S_{1}$ & $S_{2}$ & & \\
\hline 0 & $\mathrm{~S}_{1}$ & 3 & 3 & 1 & 0 & 2 & 0.67 \\
\hline 0 & $S_{2}$ & O & 10 & 0 & 1 & 9 & 0.5 \\
\hline & $C_{j}-E_{j}$ & $\begin{array}{l}3 \\
4\end{array}$ & -2 & 0 & 0 & 0 & \\
\hline 0 & $S_{1}$ & 0 & $4 / 3$ & 1 & $-1 / 6$ & $1 / 2$ & \\
\hline 3 & $\mathrm{Y}_{1}$ & 1 & $5 / 9$ & 0 & $1 / 18$ & $1 / 2$ & \\
\hline & $\mathrm{C}_{\mathrm{j}}-\mathrm{E}_{\mathrm{j}}$ & 0 & $-11 / 3$ & 0 & $-1 / 6$ & $3 / 2$ & \\
\hline
\end{tabular}

Table I

Thus $y_{1}=1 / 2, \quad y_{2}=0$

Now $\left(x_{1}, x_{2}\right)=\frac{\left(y_{1}, y_{2}\right) \beta}{1-d\left(y_{1}, y_{2}\right)}=\frac{(1 / 2,0) .1}{1-(11)(1 / 2,0)}=\frac{(1 / 2,0)}{1-1 / 2}=\frac{(1 / 2,0)}{1 / 2}=(1,0)$

Thus $\max \mathrm{Z}_{1}=3 / 2$ with $\mathrm{x}_{1}=1, \mathrm{x}_{2}=0$

Second objective function in equation (1.8),

$$
\max z_{2}=\frac{9 x_{1}+3 x_{2}}{x_{1}+x_{2}+1} \quad \begin{gathered}
x_{1}+x_{2} \leq 2 \\
\mathrm{~s} / \mathrm{t} 9 x_{1}+x_{2} \leq 9 \\
x_{1}, x_{2} \geq 0
\end{gathered}
$$

So, $c=(9,3), d=(1,1), \alpha=0, \beta=1, A_{1}=(1,1), A_{2}=(9,1), b_{1}=2, b_{2}=9$

$\max \quad H(y)=[I y]+j=\frac{c \beta-d \alpha}{\beta}[y]+0=(9,3) \cdot\left[\begin{array}{l}y_{1} \\ y_{2}\end{array}\right]=9 y_{1}+3 y_{2}$

For first constraint,

$$
K_{1}=(1,1) \cdot 1+2(1,1)=(1,1)+(2,2)=(3,3), K y \leq L \Rightarrow(3,3)\left(\begin{array}{l}
y_{1} \\
y_{2}
\end{array}\right) \leq 2 \Rightarrow 3 y_{1}+3 y_{2} \leq 2
$$

For second constraint,

$$
K_{2}=(9,1) \cdot 1+9(1,1)=(9,1)+(9,9)=(18,10), K y \leq L \Rightarrow(18,10)\left(\begin{array}{l}
y_{1} \\
y_{2}
\end{array}\right) \leq 9 \Rightarrow 18 y_{1}+10 y_{2} \leq 9
$$




$$
\begin{gathered}
\max , H(y)=9 y_{1}+3 y_{2} \\
s / t 3 y_{1}+3 y_{2} \leq 2 \\
18 y_{1}+10 y_{2} \leq 9 \\
y_{1}, y_{2} \geq 0
\end{gathered} \Leftrightarrow \begin{array}{r}
\max H=9 y_{1}+3 y_{2} \\
s / t 3 y_{1}+3 y_{2}+s_{1}=2 \\
18 y_{1}+10 y_{2}+s_{2}=9
\end{array}
$$

\begin{tabular}{|c|c|c|c|c|c|c|c|}
\hline \multirow[t]{2}{*}{$c_{B}$} & \multirow{2}{*}{ Basis ${ }^{c_{j}}$} & 9 & 3 & 0 & 0 & & \\
\hline & & $\mathrm{Y}_{1}$ & $Y_{2}$ & $S_{1}$ & $S_{2}$ & & \\
\hline 0 & $\mathrm{~S}_{1}$ & 3 & 3 & 1 & 0 & 2 & 0.667 \\
\hline 0 & $\mathrm{~S}_{2}$ & 10 & 10 & 0 & 1 & 9 & 0.5 \\
\hline & $C_{j}-E_{j}$ & $\begin{array}{l}9 \\
\uparrow\end{array}$ & 3 & 0 & 0 & 0 & \\
\hline 0 & $S_{1}$ & 0 & $4 / 3$ & 1 & $-1 / 6$ & $1 / 2$ & \\
\hline 9 & $Y_{1}$ & 1 & $5 / 9$ & 0 & $1 / 18$ & $1 / 2$ & \\
\hline & $C_{j}-E_{j}$ & 0 & -2 & 0 & $-1 / 2$ & $9 / 2$ & \\
\hline
\end{tabular}

Table II

Thus $y_{1}=1 / 2, \quad y_{2}=0$

Now $\left(x_{1}, x_{2}\right)=\frac{\left(y_{1}, y_{2}\right) \beta}{1-d\left(y_{1}, y_{2}\right)}=\frac{(1 / 2,0) .1}{1-(1,1)(1 / 2,0)}=\frac{(1 / 2,0)}{1-1 / 2}=\frac{(1 / 2,0)}{1 / 2}=(1,0)$

Thus $\max Z_{2}=9 / 2$ with $x_{1}=1, x_{2}=0$

For third objective function from equation (1.8)

$$
\max z_{3}=\frac{3 x_{1}-5 x_{2}}{2 x_{1}+2 x_{2}+2} \quad \begin{gathered}
x_{1}+x_{2} \leq 2 \\
\text { s/t } 9 x_{1}+x_{2} \leq 9 \\
x_{1}, x_{2} \geq 0
\end{gathered}
$$

So, $c=(3,-5), d=(2,2), \alpha=0, \beta=2, A_{1}=(1,1), A_{2}=(9,1), b_{1}=2, b_{2}=9$

$\max \quad H(y)=[I y]+j=\frac{c \beta-d \alpha}{\beta}[y]+0=(3,-5) \cdot\left[\begin{array}{l}y_{1} \\ y_{2}\end{array}\right]=3 y_{1}-5 y_{2}$

For first constraint,

$$
\begin{gathered}
K_{1}=(1,1) \cdot 2+2(2,2)=(2,2)+(4,4)=(6,6), \\
K y \leq L \Rightarrow(6,6)\left(\begin{array}{l}
y_{1} \\
y_{2}
\end{array}\right) \leq 2 \Rightarrow 6 y_{1}+6 y_{2} \leq 2
\end{gathered}
$$

For second constraint,

$$
\begin{aligned}
& K_{2}=(9,1) \cdot 2+9(2,2)=(18,2)+(18,18)=(36,20) \\
& K y \leq L \Rightarrow(36,20)\left(\begin{array}{l}
y_{1} \\
y_{2}
\end{array}\right) \leq 9 \Rightarrow 36 y_{1}+20 y_{2} \leq 9 \\
& \max , H(y)=3 y_{1}-5 y_{2} \quad \max H=3 y_{1}-5 y_{2} \\
& \text { Thus }
\end{aligned}
$$

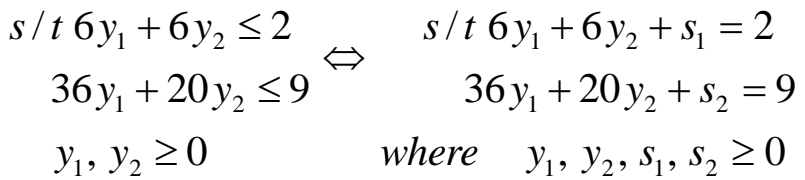


Thus $y_{1}=1 / 4, \quad y_{2}=0$

\begin{tabular}{|c|c|c|c|c|c|c|c|}
\hline \multicolumn{8}{|c|}{ Table III } \\
\hline \multirow[t]{2}{*}{$c_{B}$} & \multirow{2}{*}{ Basis ${ }^{c_{j}}$} & 3 & -5 & 0 & 0 & & \\
\hline & & $Y_{1}$ & $\mathrm{Y}_{2}$ & $S_{1}$ & $\mathrm{~S}_{2}$ & & \\
\hline 0 & $\mathrm{~S}_{1}$ & 6 & 6 & 1 & 0 & 2 & 0.33 \\
\hline 0 & $\mathrm{~S}_{2}$ & 36 & 20 & 0 & 1 & 9 & 0.25 \\
\hline & $C_{j}-E_{j}$ & $\begin{array}{l}3 \\
4\end{array}$ & -5 & 0 & 0 & 0 & \\
\hline 0 & $S_{1}$ & 0 & $8 / 3$ & 1 & $-1 / 6$ & $1 / 2$ & \\
\hline 3 & $\mathrm{Y}_{1}$ & 1 & $5 / 9$ & 0 & $1 / 36$ & $1 / 4$ & \\
\hline & $\mathrm{C}_{\mathrm{j}}-\mathrm{E}_{\mathrm{j}}$ & 0 & $-20 / 3$ & 0 & $-1 / 2$ & $3 / 4$ & \\
\hline
\end{tabular}

Now $\left(x_{1}, x_{2}\right)=\frac{\left(y_{1}, y_{2}\right) \beta}{1-d\left(y_{1}, y_{2}\right)}=\frac{(1 / 4,0) .2}{1-(2,2)(1 / 4,0)}=\frac{(1 / 2,0)}{1-1 / 2}=\frac{(1 / 2,0)}{1 / 2}=(1,0)$

Thus $\max \mathrm{Z}_{3}=3 / 4$ with $\mathrm{x}_{1}=1, \mathrm{x}_{2}=0$

For fourth objective function from equation (1.8)

$$
\min \quad z_{4}=\frac{-6 x_{1}+2 x_{2}}{2 x_{1}+2 x_{2}+2} \quad \begin{gathered}
x_{1}+x_{2} \leq 2 \\
\text { s/t } 9 x_{1}+x_{2} \leq 9 \\
x_{1}, x_{2} \geq 0
\end{gathered}
$$

So, $c=(-6,2), d=(2,2), \alpha=0, \beta=2, A_{1}=(1,1), A_{2}=(9,1), b_{1}=2, b_{2}=9$

$\min H(y)=[I y]+j=\frac{c \beta-d \alpha}{\beta}[y]+0=(-6,2) \cdot\left[\begin{array}{l}y_{1} \\ y_{2}\end{array}\right]=-6 y_{1}+2 y_{2}$

For first constraint,

$$
K_{1}=(1,1) \cdot 2+2(2,2)=(2,2)+(4,4)=(6,6), K y \leq L \Rightarrow(6,6)\left(\begin{array}{l}
y_{1} \\
y_{2}
\end{array}\right) \leq 2 \Rightarrow 6 y_{1}+6 y_{2} \leq 2
$$

For second constraint,

\begin{tabular}{|c|c|c|c|c|c|c|c|}
\hline \multicolumn{8}{|c|}{ Table IV } \\
\hline$c_{B}$ & $c_{j}$ & 6 & -2 & 0 & 0 & & \\
\hline & Basis & $\mathrm{Y}_{1}$ & $\mathrm{Y}_{2}$ & $S_{1}$ & $\mathrm{~S}_{2}$ & & \\
\hline 0 & $\mathrm{~S}_{1}$ & 6 & 6 & 1 & 0 & 2 & 0.33 \\
\hline 0 & $\mathrm{~S}_{2}$ & & 20 & 0 & 1 & 9 & 0.25 \\
\hline & $C_{j}-E_{j}$ & 6 & -2 & 0 & 0 & 0 & \\
\hline 0 & $S_{1}$ & 0 & $8 / 3$ & 1 & $-1 / 6$ & $1 / 2$ & \\
\hline 3 & $\mathrm{Y}_{1}$ & 1 & $5 / 9$ & 0 & $1 / 36$ & $1 / 4$ & \\
\hline & $\mathrm{C}_{\mathrm{j}}-\mathrm{E}_{\mathrm{j}}$ & 0 & $-16 / 3$ & 0 & $-1 / 6$ & $3 / 2$ & \\
\hline
\end{tabular}

$$
\begin{aligned}
& K_{2}=(9,1) \cdot 2+9(2,2)=(18,2)+(18,18)=(36,20) \\
& K y \leq L \Rightarrow(36,20)\left(\begin{array}{l}
y_{1} \\
y_{2}
\end{array}\right) \leq 9 \Rightarrow 36 y_{1}+20 y_{2} \leq 9 \\
& \max , H(y)=6 y_{1}-2 y_{2} \quad \max H=6 y_{1}-2 y_{2} \\
& s / t 6 y_{1}+6 y_{2} \leq 2 \Leftrightarrow s / t 6 y_{1}+6 y_{2}+s_{1}=2 \\
& 36 y_{1}+20 y_{2} \leq 9 \Leftrightarrow \quad 36 y_{1}+20 y_{2}+s_{2}=9 \\
& y_{1}, y_{2} \geq 0 \quad \text { where } y_{1}, y_{2}, s_{1}, s_{2} \geq 0
\end{aligned}
$$


Thus $y_{1}=1 / 4, \quad y_{2}=0$

Now $\left(x_{1}, x_{2}\right)=\frac{\left(y_{1}, y_{2}\right) \beta}{1-d\left(y_{1}, y_{2}\right)}=\frac{(1 / 4,0) \cdot 2}{1-(2,2)(1 / 4,0)}=\frac{(1 / 2,0)}{1-1 / 2}=\frac{(1 / 2,0)}{1 / 2}=(1,0)$

Thus $\max \mathrm{Z}_{4}=-3 / 2$ with $\mathrm{x}_{1}=1, \mathrm{x}_{2}=0$

For fifth objective function from equation (1.8)

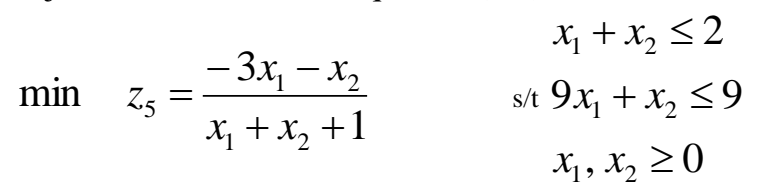

So, $c=(-3,-1), d=(1,1), \alpha=0, \beta=1, A_{1}=(1,1), A_{2}=(9,1), b_{1}=2, b_{2}=9$

$\min H(y)=[I y]+j=\frac{c \beta-d \alpha}{\beta}[y]+0=(-3,-1) \cdot\left[\begin{array}{l}y_{1} \\ y_{2}\end{array}\right]=-3 y_{1}-y_{2}$

For first constraint,

$$
K_{1}=(1,1) \cdot 1+2(1,1)=(1,1)+(2,2)=(3,3), K y \leq L \Rightarrow(3,3)\left(\begin{array}{l}
y_{1} \\
y_{2}
\end{array}\right) \leq 2 \Rightarrow 3 y_{1}+3 y_{2} \leq 2
$$

For second constraint,

$$
\begin{aligned}
& K_{2}=(9,1) \cdot 1+9(1,1)=(9,1)+(9,9)=(18,10), K y \leq L \Rightarrow(18,10)\left(\begin{array}{l}
y_{1} \\
y_{2}
\end{array}\right) \leq 9 \Rightarrow 18 y_{1}+10 y_{2} \leq 9 \\
& \max , H(y)=3 y_{1}+y_{2} \quad \max H=3 y_{1}+y_{2} \\
& s / t 3 y_{1}+3 y_{2} \leq 2 \Leftrightarrow s / t 3 y_{1}+3 y_{2}+s_{1}=2 \\
& 18 y_{1}+10 y_{2} \leq 9 \Leftrightarrow \quad 18 y_{1}+10 y_{2}+s_{2}=9 \\
& y_{1}, y_{2} \geq 0 \quad \text { where } y_{1}, y_{2}, s_{1}, s_{2} \geq 0
\end{aligned}
$$

Thus $y_{1}=1 / 2, \quad y_{2}=0$

Now $\left(x_{1}, x_{2}\right)=\frac{\left(y_{1}, y_{2}\right) \beta}{1-d\left(y_{1}, y_{2}\right)}=\frac{(1 / 2,0) .1}{1-(1,1)(1 / 2,0)}=\frac{(1 / 2,0)}{1-1 / 2}=\frac{(1 / 2,0)}{1 / 2}=(1,0)$

Thus $\max Z_{5}=-3 / 2$ with $x_{1}=1, x_{2}=0$

The values obtained from the objective functions are summarized in Table VI.

Table VI

\begin{tabular}{|c|c|c|c|c|}
\hline $\mathrm{I}$ & $\varphi_{i}$ & $\mathrm{x}_{\mathrm{i}}$ & $\mathrm{AA}_{\mathrm{i}}=\left|\varphi_{i}\right|$ & $\mathrm{AL}_{\mathrm{i}}=\left|\varphi_{i}\right|$ \\
\hline 1 & $3 / 2$ & $(1,0)$ & $3 / 2$ & \\
\hline 2 & $9 / 2$ & $(1,0)$ & $9 / 2$ & \\
\hline 3 & $3 / 4$ & $(1,0)$ & $3 / 4$ & $3 / 2$ \\
\hline 4 & $-3 / 2$ & $(1,0)$ & & $3 / 2$ \\
\hline 5 & $-3 / 2$ & $(1,0)$ & & \\
\hline
\end{tabular}

Now, by Chandra Sen's approach:

$\operatorname{Max} \mathrm{Z}=\sum_{k=1}^{r} \frac{z_{k}}{\left|\varphi_{k}\right|}-\sum_{k=r+1}^{s} \frac{z_{k}}{\left|\varphi_{k}\right|}$ 


$$
\begin{aligned}
& =\frac{2}{3} \frac{3 x_{1}-2 x_{2}}{x_{1}+x_{2}+1}+\frac{2}{9} \frac{9 x_{1}+3 x_{2}}{x_{1}+x_{2}+1}+\frac{4}{3} \frac{3 x_{1}-5 x_{2}}{2 x_{1}+2 x_{2}+2}-\left[\frac{2}{3} \frac{-6 x_{1}+2 x_{2}}{2 x_{1}+2 x_{2}+2}+\frac{2}{3} \frac{-3 x_{1}-x_{2}}{x_{1}+x_{2}+1}\right] \\
& =\frac{1}{x_{1}+x_{2}+1}\left[x_{1}(2+2+2)+x_{2}(2 / 3-10 / 3-4 / 3)\right]-\frac{1}{x_{1}+x_{2}+1}\left[-4 x_{1}\right]=\frac{10 x_{1}-4 x_{2}}{x_{1}+x_{2}+1}
\end{aligned}
$$

So the single objective function is

$$
\max , z=\frac{10 x_{1}-4 x_{2}}{x_{1}+x_{2}+1} \quad \begin{aligned}
& x_{1}+x_{2} \leq 2 \\
& 9 x_{1}+x_{2} \leq 9 \\
& \text { s/t } x_{1}, x_{2} \geq 0
\end{aligned}
$$

Again the equation (1.10) is fractional, so we have to use modified simplex method to find its solution. So from modified simplex method described in equation (1.9) we get

$c=(10,4), d=(1,1), \alpha=0, \beta=1, A_{1}=(1,1), A_{2}=(9,1), b_{1}=2, b_{2}=9$

$\max \quad H(y)=[I y]+j=\frac{c \beta-d \alpha}{\beta}[y]+0=(10,4) \cdot\left[\begin{array}{l}y_{1} \\ y_{2}\end{array}\right]=10 y_{1}+4 y_{2}$

For first constraint,

$$
\begin{aligned}
& K_{1}=(1,1) .1+2(1,1)=(1,1)+(2,2)=(3,3), \\
& K y \leq L \Rightarrow(3,3)\left(\begin{array}{l}
y_{1} \\
y_{2}
\end{array}\right) \leq 2 \Rightarrow 3 y_{1}+3 y_{2} \leq 2
\end{aligned}
$$

\begin{tabular}{|c|c|c|c|c|c|c|c|}
\hline \multicolumn{8}{|c|}{ Table VII } \\
\hline \multirow[t]{2}{*}{$\mathrm{c}_{\mathrm{B}}$} & \multirow{2}{*}{ Basis ${ }^{c_{j}}$} & 10 & 4 & 0 & 0 & & \\
\hline & & $Y_{1}$ & $\mathrm{Y}_{2}$ & $S_{1}$ & $\mathrm{~S}_{2}$ & & \\
\hline 0 & $\mathrm{~S}_{1}$ & 3 & 3 & 1 & 0 & 2 & 0.67 \\
\hline \multirow[t]{2}{*}{0} & $\mathrm{~S}_{2}$ & & 10 & 0 & 1 & 9 & 0.5 \\
\hline & $C_{j}-E_{j}$ & 10 & 4 & 0 & 0 & 0 & \\
\hline 0 & $S_{1}$ & 0 & $4 / 3$ & 1 & $-1 / 6$ & $1 / 2$ & \\
\hline \multirow[t]{2}{*}{10} & $\mathrm{Y}_{1}$ & 1 & $5 / 9$ & 0 & $1 / 18$ & $1 / 2$ & \\
\hline & $C_{j}-E_{j}$ & 0 & $-14 / 9$ & 0 & $-5 / 9$ & $5 / 2$ & \\
\hline
\end{tabular}

For second constraint,

$$
\begin{aligned}
& K_{2}=(9,1) \cdot 1+9(1,1)=(9,1)+(9,9)=(18,10) \\
& K y \leq L \Rightarrow(18,10)\left(\begin{array}{l}
y_{1} \\
y_{2}
\end{array}\right) \leq 9 \Rightarrow 18 y_{1}+10 y_{2} \leq 9 \\
& \max , H(y)=10 y_{1}+4 y_{2} \quad \max H=10 y_{1}+4 y_{2} \\
& s / t 3 y_{1}+3 y_{2} \leq 2 \Leftrightarrow \quad s / t 3 y_{1}+3 y_{2}+s_{1}=2 \\
& 18 y_{1}+10 y_{2} \leq 9 \Leftrightarrow \quad 18 y_{1}+10 y_{2}+s_{2}=9 \\
& y_{1}, y_{2} \geq 0 \quad \text { where } y_{1}, y_{2}, s_{1}, s_{2} \geq 0
\end{aligned}
$$

Thus $y_{1}=1 / 2, \quad y_{2}=0$

Now $\left(x_{1}, x_{2}\right)=\frac{\left(y_{1}, y_{2}\right) \beta}{1-d\left(y_{1}, y_{2}\right)}=\frac{(1 / 2,0) .1}{1-(1,1)(1 / 2,0)}=\frac{(1 / 2,0)}{1-1 / 2}=\frac{(1 / 2,0)}{1 / 2}=(1,0)$

Thus $\max \mathrm{Z}=5$ with $\mathrm{x}_{1}=1, \mathrm{x}_{2}=0$

From Table VI, we get

$$
\text { A.M. }(3 / 2,9 / 2,3 / 4)=9 / 4, \quad \text { A.M. }(3 / 2,3 / 2)=3 / 2
$$

So, by using equation (1.4) we have

$$
\text { Max Z }=\sum_{i=1}^{r} \frac{z_{i}}{A \cdot M\left(A A_{i}\right)}-\sum_{i=r+1}^{s} \frac{z_{i}}{A \cdot M\left(A L_{i}\right)}
$$




$$
\begin{aligned}
\operatorname{Max} z= & \frac{4}{9}\left[\frac{3 x_{1}-2 x_{2}}{x_{1}+x_{2}+1}+\frac{9 x_{1}+3 x_{2}}{x_{1}+x_{2}+1}+\frac{3 x_{1}-5 x_{2}}{2 x_{1}+2 x_{2}+2}\right]-\frac{2}{3}\left[\frac{-6 x_{1}+2 x_{2}}{2 x_{1}+2 x_{2}+2}+\frac{-3 x_{1}-x_{2}}{x_{1}+x_{2}+1}\right] \\
& =\frac{10 x_{1}-2 / 3 x_{2}}{x_{1}+x_{2}+1}
\end{aligned}
$$

Thus $\max , z=\frac{10 x_{1}-2 / 3 x_{2}}{x_{1}+x_{2}+1}$

$$
\begin{aligned}
& x_{1}+x_{2} \leq 2 \\
& 9 x_{1}+x_{2} \leq 9 \\
& \text { s/t } x_{1}, x_{2} \geq 0
\end{aligned}
$$

$c=(10,-2 / 3), d=(1,1), \alpha=0, \beta=1, A_{1}=(1,1), A_{2}=(9,1), b_{1}=2, b_{2}=9$

$\max H(y)=[I y]+j=\frac{c \beta-d \alpha}{\beta}[y]+0=(10,-2 / 3) \cdot\left[\begin{array}{l}y_{1} \\ y_{2}\end{array}\right]=10 y_{1}-2 / 3 y_{2}$

For first constraint,

$$
K_{1}=(1,1) \cdot 1+2(1,1)=(1,1)+(2,2)=(3,3), K y \leq L \Rightarrow(3,3)\left(\begin{array}{l}
y_{1} \\
y_{2}
\end{array}\right) \leq 2 \Rightarrow 3 y_{1}+3 y_{2} \leq 2
$$

For second constraint,

$$
K_{2}=(9,1) \cdot 1+9(1,1)=(9,1)+(9,9)=(18,10), K y \leq L \Rightarrow(18,10)\left(\begin{array}{l}
y_{1} \\
y_{2}
\end{array}\right) \leq 9 \Rightarrow 18 y_{1}+10 y_{2} \leq 9
$$

$\max , H(y)=10 y_{1}-2 / 3 y_{2} \quad \max H=10 y_{1}-2 / 3 y_{2}$

$$
\text { Thus } \begin{gathered}
s / t 3 y_{1}+3 y_{2} \leq 2 \\
18 y_{1}+10 y_{2} \leq 9 \\
y_{1}, y_{2} \geq 0
\end{gathered} \Leftrightarrow \begin{array}{r}
s / t 3 y_{1}+3 y_{2}+s_{1}=2 \\
18 y_{1}+10 y_{2}+s_{2}=9 \\
\text { where } \quad y_{1}, y_{2}, s_{1}, s_{2} \geq 0
\end{array}
$$

\begin{tabular}{|c|c|c|c|c|c|c|c|}
\multicolumn{7}{c|}{ Table VIII } \\
\hline $\mathrm{c}_{\mathrm{B}}$ & \multirow{3}{*}{$\mathrm{c}_{\mathrm{j}}$} & 10 & $-2 / 3$ & 0 & 0 & & \\
\cline { 2 - 9 } & $\mathrm{S}_{1}$ & $\mathrm{Y}_{2}$ & $\mathrm{~S}_{1}$ & $\mathrm{~S}_{2}$ & & \\
\hline 0 & $\mathrm{~S}_{1}$ & 3 & 3 & 1 & 0 & 2 & 0.67 \\
\hline 0 & $\mathrm{~S}_{2}$ & 18 & 10 & 0 & 1 & 9 & 0.5 \\
& & & & & & & \\
\hline & $\mathrm{C}_{\mathrm{j}-\mathrm{E}_{\mathrm{j}}}$ & 10 & $-2 / 3$ & 0 & 0 & 0 & \\
& & $\uparrow$ & & & & & \\
\hline 0 & $\mathrm{~S}_{1}$ & 0 & $4 / 3$ & 1 & $-1 / 6$ & $1 / 2$ & \\
\hline 10 & $\mathrm{Y}_{1}$ & 1 & $5 / 9$ & 0 & $1 / 18$ & $1 / 2$ & \\
\hline & $\mathrm{C}_{\mathrm{j}}-\mathrm{E}_{\mathrm{j}}$ & 0 & $-56 / 9$ & 0 & $-5 / 9$ & $-5 / 9$ & \\
\hline
\end{tabular}

Thus $y_{1}=1 / 2, \quad y_{2}=0$

Now $\left(x_{1}, x_{2}\right)=\frac{\left(y_{1}, y_{2}\right) \beta}{1-d\left(y_{1}, y_{2}\right)}=\frac{(1 / 2,0) .1}{1-(1,1)(1 / 2,0)}=\frac{(1 / 2,0)}{1-1 / 2}=\frac{(1 / 2,0)}{1 / 2}=(1,0)$

Thus $\max \mathrm{Z}=5$ with $\mathrm{x}_{1}=1, \mathrm{x}_{2}=0$

From Table VI, we get G.M. $(3 / 2,9 / 2,3 / 4)=1.7171 ;$ G.M. $(3 / 2,3 / 2)=1.5$

So, by using equation (1.5) we have

$\operatorname{Max} \mathrm{Z}=\sum_{i=1}^{r} \frac{z_{i}}{G \cdot M\left(A A_{i}\right)}-\sum_{i=r+1}^{s} \frac{z_{i}}{G \cdot M\left(A L_{i}\right)}$

$\operatorname{Max} z=\frac{1}{1.7171}\left[\frac{3 x_{1}-2 x_{2}}{x_{1}+x_{2}+1}+\frac{9 x_{1}+3 x_{2}}{x_{1}+x_{2}+1}+\frac{3 x_{1}-5 x_{2}}{2 x_{1}+2 x_{2}+2}\right]-\frac{1}{1.5}\left[\frac{-6 x_{1}+2 x_{2}}{2 x_{1}+2 x_{2}+2}+\frac{-3 x_{1}-x_{2}}{x_{1}+x_{2}+1}\right]$

$$
=\frac{11.862 x_{1}-0.8736 x_{2}}{x_{1}+x_{2}+1}
$$


Thus $\max Z=\frac{11.862 x_{1}-0.8736 x_{2}}{x_{1}+x_{2}+1} \quad \begin{aligned} & x_{1}+x_{2} \leq 2 \\ & 9 x_{1}+x_{2} \leq 9 \\ & \text { s/t } x_{1}, x_{2} \geq 0\end{aligned}$

$c=(11.862,-0.8736), d=(1,1), \alpha=0, \beta=1, A_{1}=(1,1), A_{2}=(9,1), b_{1}=2, b_{2}=9$

$\max \quad H(y)=[I y]+j=\frac{c \beta-d \alpha}{\beta}[y]+0=(11.862,-0.8736) .\left[\begin{array}{l}y_{1} \\ y_{2}\end{array}\right]=11.862 y_{1}-0.8736 y_{2}$

For first constraint,

$K_{1}=(1,1) \cdot 1+2(1,1)=(1,1)+(2,2)=(3,3), K y \leq L \Rightarrow(3,3)\left(\begin{array}{l}y_{1} \\ y_{2}\end{array}\right) \leq 2 \Rightarrow 3 y_{1}+3 y_{2} \leq 2$

For second constraint

$$
K_{2}=(9,1) \cdot 1+9(1,1)=(9,1)+(9,9)=(18,10), K y \leq L \Rightarrow(18,10)\left(\begin{array}{l}
y_{1} \\
y_{2}
\end{array}\right) 9 \Rightarrow 18 y_{1}+10 y_{2} \leq 9
$$

$\max , H(y)=11.862 y_{1}-0.8736 y_{2} \quad \max H=11.862 y_{1}-0.8736 y_{2}$

Thus

$$
\begin{gathered}
s / t 3 y_{1}+3 y_{2} \leq 2 \\
18 y_{1}+10 y_{2} \leq 9 \\
y_{1}, y_{2} \geq 0
\end{gathered} \Leftrightarrow \begin{array}{r}
s / t 3 y_{1}+3 y_{2}+s_{1}=2 \\
18 y_{1}+10 y_{2}+s_{2}=9 \\
\text { where } \quad y_{1}, y_{2}, s_{1}, s_{2} \geq 0
\end{array}
$$

\begin{tabular}{|c|c|c|c|c|c|c|c|}
\hline \multicolumn{8}{|c|}{ Table IX } \\
\hline \multirow[t]{2}{*}{$\mathrm{c}_{\mathrm{B}}$} & \multirow{2}{*}{ Basis ${ }^{c_{j}}$} & 11.862 & -0.8736 & 0 & 0 & & \\
\hline & & $\mathrm{Y}_{1}$ & $\mathrm{Y}_{2}$ & $\overline{S_{1}}$ & $\mathrm{~S}_{2}$ & & \\
\hline 0 & $\mathrm{~S}_{1}$ & 3 & 3 & 1 & 0 & 2 & 0.67 \\
\hline \multirow[t]{2}{*}{0} & $\mathrm{~S}_{2}$ & & 10 & 0 & 1 & 9 & 0.5 \\
\hline & $C_{j}-E_{j}$ & 11.862 & -0.8736 & 0 & 0 & 0 & \\
\hline 0 & $S_{1}$ & 0 & $4 / 3$ & 1 & $-1 / 6$ & $1 / 2$ & \\
\hline \multirow[t]{2}{*}{11.862} & $\mathrm{Y}_{1}$ & 1 & $5 / 9$ & 0 & $1 / 18$ & $1 / 2$ & \\
\hline & $\mathrm{C}_{\mathrm{j}}-\mathrm{E}_{\mathrm{j}}$ & 0 & -7.4636 & 0 & -0.659 & -5.931 & \\
\hline
\end{tabular}

Thus $y_{1}=1 / 2, \quad y_{2}=0$

Now $\left(x_{1}, x_{2}\right)=\frac{\left(y_{1}, y_{2}\right) \beta}{1-d\left(y_{1}, y_{2}\right)}=\frac{(1 / 2,0) .1}{1-(1,1)(1 / 2,0)}=\frac{(1 / 2,0)}{1-1 / 2}=\frac{(1 / 2,0)}{1 / 2}=(1,0)$

Thus $\max \mathrm{Z}=5.931$ with $\mathrm{x}_{1}=1, \mathrm{x}_{2}=0$

New Arithmetic average technique:

Let $\mathrm{m}_{1}=0.75, \mathrm{~m}_{2}=1.5 ; \frac{m_{1}+m_{2}}{2}=\frac{0.75+1.5}{2}=1.125$

$\max \quad z=\left(\sum_{i=1}^{r} z_{i}-\sum_{i=r+1}^{s} z_{i}\right) / A . A v=\frac{39 x_{1}-3 x_{2}}{2(1.125)\left(x_{1}+x_{2}+1\right)}=\frac{39 x_{1}-3 x_{2}}{2.25 .\left(x_{1}+x_{2}+1\right)}=\frac{17.33 x_{1}-1.33 x_{2}}{x_{1}+x_{2}+1}$

Thus $\max Z=\frac{17.33 x_{1}-1.33 x_{2}}{x_{1}+x_{2}+1}$

$$
\begin{gathered}
x_{1}+x_{2} \leq 2 \\
9 x_{1}+x_{2} \leq 9 \\
\mathrm{~s} / \mathrm{t} x_{1}, x_{2} \geq 0
\end{gathered}
$$

$c=(17.33,-1.33), d=(1,1), \alpha=0, \beta=1, A_{1}=(1,1), A_{2}=(9,1), b_{1}=2, b_{2}=9$

$\max \quad H(y)=[I y]+j=\frac{c \beta-d \alpha}{\beta}[y]+0=(17.33,-1.33) \cdot\left[\begin{array}{l}y_{1} \\ y_{2}\end{array}\right]=17.33 y_{1}-1.33 y_{2}$

For first constraint, 


$$
K_{1}=(1,1) \cdot 1+2(1,1)=(1,1)+(2,2)=(3,3), K y \leq L \Rightarrow(3,3)\left(\begin{array}{l}
y_{1} \\
y_{2}
\end{array}\right) \leq 2 \Rightarrow 3 y_{1}+3 y_{2} \leq 2
$$

For second constraint,

$$
\begin{gathered}
K_{2}=(9,1) .1+9(1,1)=(9,1)+(9,9)=(18,10), K y \leq L \Rightarrow(18,10)\left(\begin{array}{l}
y_{1} \\
y_{2}
\end{array}\right) \leq 9 \Rightarrow 18 y_{1}+10 y_{2} \leq 9 \\
\begin{array}{ccc}
\max , H(y)=17.33 y_{1}-1.33 y_{2} & \max H=17.33 y_{1}-1.33 y_{2}
\end{array} \\
\begin{array}{ccc}
s / t 3 y_{1}+3 y_{2} \leq 2 & s / t 3 y_{1}+3 y_{2}+s_{1}=2 \\
18 y_{1}+10 y_{2} \leq 9 & 18 y_{1}+10 y_{2}+s_{2}=9 \\
y_{1}, y_{2} \geq 0 & \text { where } y_{1}, y_{2}, s_{1}, s_{2} \geq 0
\end{array}
\end{gathered}
$$

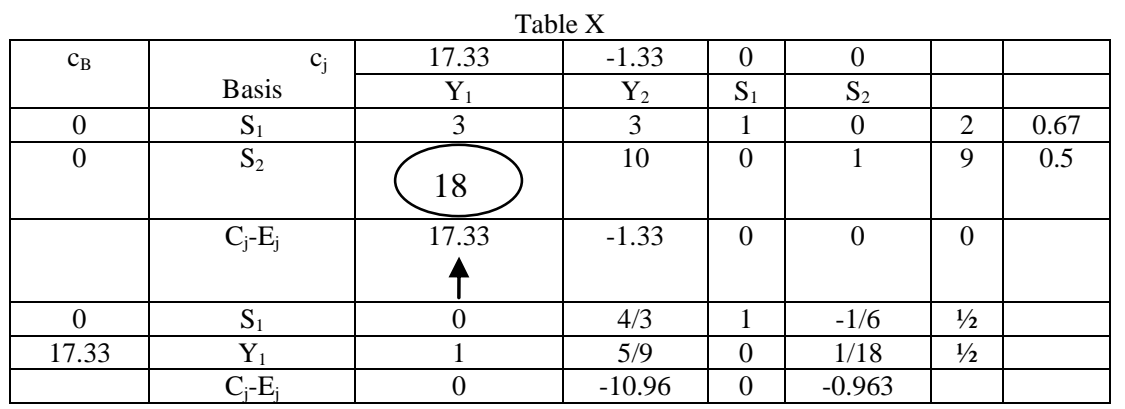

Thus $y_{1}=1 / 2, \quad y_{2}=0$

Now $\left(x_{1}, x_{2}\right)=\frac{\left(y_{1}, y_{2}\right) \beta}{1-d\left(y_{1}, y_{2}\right)}=\frac{(1 / 2,0) .1}{1-(1,1)(1 / 2,0)}=\frac{(1 / 2,0)}{1-1 / 2}=\frac{(1 / 2,0)}{1 / 2}=(1,0)$

Thus $\max \mathrm{Z}=8.665$ with $\mathrm{x}_{1}=1, \mathrm{x}_{2}=0$

\section{New Geometric average technique:}

Let $\mathrm{m}_{1}=0.75, \mathrm{~m}_{2}=1.5$; So $G . A v=\sqrt{0.75(1.5)}=1.061$

$\max \quad z=\left(\sum_{i=1}^{r} z_{i}-\sum_{i=r+1}^{s} z_{i}\right) / G . A v$

$$
=\frac{39 x_{1}-3 x_{2}}{2(1.061)\left(x_{1}+x_{2}+1\right)}=\frac{39 x_{1}-3 x_{2}}{2.122 .\left(x_{1}+x_{2}+1\right)}=\frac{18.379 x_{1}-1.4137 x_{2}}{x_{1}+x_{2}+1}
$$

Thus maz $z=\frac{18.379 x_{1}-1.4137 x_{2}}{x_{1}+x_{2}+1} \quad \begin{aligned} & x_{1}+x_{2} \leq 2 \\ & 9 x_{1}+x_{2} \leq 9 \\ & \text { s/t } x_{1}, x_{2} \geq 0\end{aligned}$

$c=(18.379,-1.4137), d=(1,1), \alpha=0, \beta=1, A_{1}=(1,1), A_{2}=(9,1), b_{1}=2, b_{2}=9$

$\max \quad H(y)=[I y]+j=\frac{c \beta-d \alpha}{\beta}[y]+0=(18.379,-1.4137) .\left[\begin{array}{l}y_{1} \\ y_{2}\end{array}\right]=18.379 y_{1}-1.4137 y_{2}$

For first constraint,

$K_{1}=(1,1) \cdot 1+2(1,1)=(1,1)+(2,2)=(3,3), K y \leq L \Rightarrow(3,3)\left(\begin{array}{l}y_{1} \\ y_{2}\end{array}\right) \leq 2 \Rightarrow 3 y_{1}+3 y_{2} \leq 2$

For second constraint,

$$
K_{2}=(9,1) \cdot 1+9(1,1)=(9,1)+(9,9)=(18,10), K y \leq L \Rightarrow(18,10)\left(\begin{array}{l}
y_{1} \\
y_{2}
\end{array}\right) \leq 9 \Rightarrow 18 y_{1}+10 y_{2} \leq 9
$$




$$
\begin{array}{cc}
\max , H(y)=18.379 y_{1}-1.4137 y_{2} \\
s / t 3 y_{1}+3 y_{2} \leq 2 \\
18 y_{1}+10 y_{2} \leq 9 \\
y_{1}, y_{2} \geq 0
\end{array} \Leftrightarrow \begin{gathered}
\max H=18.379 y_{1}-1.4137 y_{2} \\
s / t 3 y_{1}+3 y_{2}+s_{1}=2 \\
18 y_{1}+10 y_{2}+s_{2}=9 \\
\text { Thus } \begin{array}{c}
y_{1}, y_{2}, s_{1}, s_{2} \geq 0
\end{array}
\end{gathered}
$$

Table XI

\begin{tabular}{|c|c|c|c|c|c|c|c|}
\hline \multirow{2}{*}{$\mathrm{c}_{\mathrm{B}}$} & $\mathrm{c}_{\mathrm{j}}$ & 18.379 & -1.4137 & 0 & 0 & & \\
\cline { 3 - 8 } & Basis & $\mathrm{Y}_{1}$ & $\mathrm{Y}_{2}$ & $\mathrm{~S}_{1}$ & $\mathrm{~S}_{2}$ & & \\
\hline 0 & $\mathrm{~S}_{1}$ & 3 & 3 & 1 & 0 & 2 & 0.67 \\
\hline 0 & $\mathrm{~S}_{2}$ & 18 & 10 & 0 & 1 & 9 & 0.5 \\
& & & & & & & \\
\hline & $\mathrm{C}_{\mathrm{j}-\mathrm{E}_{\mathrm{j}}}$ & $\begin{array}{c}18.379 \\
\mathrm{~T}\end{array}$ & -1.4137 & 0 & 0 & 0 & \\
& & 0 & & & & & \\
\hline 0 & $\mathrm{~S}_{1}$ & 1 & $5 / 3$ & 1 & $-1 / 6$ & $1 / 2$ & \\
\hline 18.379 & $\mathrm{Y}_{1}$ & 0 & -11.624 & 0 & -1.021 & & \\
\hline & $\mathrm{C}_{\mathrm{j}}-\mathrm{E}_{\mathrm{j}}$ & & & & & & \\
\hline
\end{tabular}

Thus $y_{1}=1 / 2, \quad y_{2}=0$

Now $\left(x_{1}, x_{2}\right)=\frac{\left(y_{1}, y_{2}\right) \beta}{1-d\left(y_{1}, y_{2}\right)}=\frac{(1 / 2,0) .1}{1-(1,1)(1 / 2,0)}=\frac{(1 / 2,0)}{1-1 / 2}=\frac{(1 / 2,0)}{1 / 2}=(1,0)$

Thus $\max Z=9.1895$ with $\mathrm{x}_{1}=1, \mathrm{x}_{2}=0$

The solutions of the MOLFPP solved by different approaches are summarized in Table XII which shows that we get the improved value of the objective function using new statistical average method proposed in this paper. So the proposed technique is justified here.

Table XII

\begin{tabular}{|c|c|c|c|c|}
\hline \multirow{2}{*}{$\begin{array}{c}\text { Chandra Sen's } \\
\text { Approach }\end{array}$} & \multicolumn{2}{|c|}{ Statistical Average Method } & \multicolumn{2}{c|}{ New Statistical Average Method } \\
\cline { 2 - 5 } & Using A.M & Using G.M & $\begin{array}{c}\text { New A. Av } \\
\text { method }\end{array}$ & $\begin{array}{c}\text { New G. Av } \\
\text { method }\end{array}$ \\
\hline Max Z=5 & Max $Z=5$ & Max $Z=5.931$ & Max $Z=8.665$ & $\begin{array}{c}\text { Max } Z=9.1895 \\
\text { with } \mathrm{x}_{1}=1, \mathrm{x}_{2}=0\end{array}$ \\
$\begin{array}{c}\text { with } \mathrm{x}_{1}=1, \\
\mathrm{x}_{2}=0\end{array}$ & $\begin{array}{c}\text { with } \mathrm{x}_{1}=1, \\
\mathrm{x}_{2}=0\end{array}$ & with $\mathrm{x}_{1}=1, \mathrm{x}_{2}=0$ & with $\mathrm{x}_{1}=1, \mathrm{x}_{2}=0$ & \\
\hline
\end{tabular}

\section{Conclusion}

In this paper, a MOLFPP has been solved using different methods such as Chandra Sen's approach and proposed statistical average method, and the results are compared in Table XII. In statistical average method, we proposed geometric and arithmetic average approach. We also proposed a new statistical average approach to solve the problem. It is observed that statistical average method results better optimization than Chandra Sen's approach of the MOLFPP. The proposed new statistical average method optimizes the problem better than that of statistical average method. We also found that geometric average technique is suited for optimizing MOLFPP better that that of arithmetic average technique.

\section{References}

[1]. Sen, Chandra., "A new approach for multi objective rural development planning”, The Indian Economic Journal, Vol.30, pp.91-96. [2] Azapagic, A., R. Clift., (1999), "Life cycle assessment and multi objective optimization", Journal of cleaner production, Vol.7, pp.135-145, 1983

[2]. Sulaiman, N.A. and Sadiq, G.W., "Solving the linear multi-objective programming problems; using mean and median value", AlRafiden Journal of Computer Sciences and Mathematics, University of Mosul, Vol.3, No. 1, pp.69-83, 2006

[3]. A. Abdil-Kadir, M.S and Sulaiman, and N.A., "An approach for multi objective fractional programming problem", Journal of the College of Education, Univ. Salahaddin, Vol. 3. pp.1-5, 1993

[4]. Sing, H.C., "Optimality condition in functional programming", Journal of Optimization Theory and Application, Vol. 33, pp.287294,1981

[5]. Sulaiman, N.A. and Othman, A.Q., "Optimal transformation Technique to solve multi-objective linear programming problem", Journal of University of Kirkuk, Vol.2. No. 2, 2007

[6]. Hamad-Amin A.O., "An adaptive Arithmetic Average Transformation Technique for solving MOLPP”, M.Sc. Thesis, University of Koya, Koya/Iraq, 2008

[7]. Nejmaddin A. Sulaiman, Rebaz B. Mustafa, "Using harmonic mean to solve multi-objective linear programming problems", American Journal of operations Research, 2016, 6, 25-30.

[8]. Wikipedia, linear programming, http://en.wikipedia.org/wiki/Linear_Programming

[9]. S. Sharma, Operation Research, Kedar Nath Ram Nath BCO. Meerut, India. (1988), 60, 559.

[10]. Operation Researh, http://www.scribd.com/doc/100903589/Operation Research, (21)

[11]. Saha, S.K., Hossain, M.R., Uddin, M.K. and Mondal, R.N. 'A New Approach of Solving Linear Fractional Programming Problem (LFP) by Using Computer Algorithm' Open Journal of Optimization, 4, 74-86, 2015 http://dx.doi.org/10.4236/ojop.2015.43010 